\title{
Metastability and Nucleation
}

Daan Frenkel

FOM Institute for Atomic and Molecular Physics, Amsterdam, NL

$\mathrm{O}^{\prime \prime}$ ne of the "grand challenges" in liquid state physics is to gain insight into activated processes on a molecular level. Why is this such a challenge? First of all: activated processes are ubiquitous and important. They include homogeneous and heterogeneous nucleation of liquids and crystals, cavitation, and conformational changes of flexible molecules. In addition, activated processes often control the rate of transport in condensed phases, e.g. diffusion of polymers in porous media or transport of defects in solids. However, in order to qualify as a challenge, a physical phenomenon should not only be important, it should also be difficult to study. In this respect, nucleation scores high. The formation of a novel phase from the metastable parent phase proceeds through the formation of a "critical nucleus". Clusters of the novel phase that are smaller than this critical size tend to disappear spontaneously - clusters that are larger than critical can continue to grow into a macroscopic domain of the novel phase. The rate of nucleation depends strongly on the free energy of the critical nucleus, while its structure and composition determine the nucleation pathway. Classical Nucleation Theory makes the assumption that nuclei of the novel phase are small spherical "droplets" that have the same properties (density, surface tension etc.) as the bulk phase.

Unfortunately, direct knowledge of the critical nucleus is limited. The problem is precisely that the formation of a critical nucleus is a fleeting event. Experiments can probe the rate at which crystallites form in a supersaturated solution, but by the time these crystallites are observed, the critical nucleus stage is long past. It is possible to estimate the size of the critical nucleus from experiment but, to my knowledge there are, at this stage, no experiments that can directly image the critical nucleus in any nucleation process

At first sight, it would seem that the problems for simulation are even worse: events that are rare in a system of 1023 particles are much rarer still in a system of only a few thousand, or even a few million, particles. Fortunately, in simulations we are not forced to wait for a rare event to happen. Using the techniques pioneered by Charles Bennett and David Chandler [1], we can make them happen and still get an accurate estimate for the nucleation rate in an unperturbed system. Using this approach, it is now possible to simulate the early stages of homogeneous (or heterogeneous) nucleation. The simulations that now start appearing $[2,3]$ reveal that the structure and free energy of the critical nucleus often deviate significantly from the predictions of Classical Nucleation Theory. It seems likely that further study, both numerical and experimental, will help us to understand how to control (enhance/suppress) nucleation of specific phases or, more generally, how to steer activated processes.

[1] see e.g.:D. Chandler, Introduction to Modern Statistical Mechanics, Oxford UP, 1987.

[2] P.R. ten Wolde et al. , Phys. Rev. Lett. 75:2714(1995), ibid. 81:3695(1998)

[3] P. R. ten Wolde and Daan Frenkel, Science 277:1975(1997). 